\title{
Breakout prediction based on particle swarm optimization back propagation neural network in continuous casting process
}

\author{
ZHANG Benguo ${ }^{1, a}$, ZHANG Xinjiang ${ }^{1, b}$ and FAN Lifeng ${ }^{2 c^{*}}$ \\ ${ }^{1}$ Yancheng institute technology, Yancheng 264005, China \\ ${ }^{2}$ Inner Mongolia University, Hohhot, 010070, China \\ azhangbenguo@163.com, bhangxinjiang1983@163.com, ${ }^{\mathrm{c}} 251155328 @ q q . c o m$ \\ *Corresponding author.
}

\begin{abstract}
Keywords: Particle swarm optimization, BP neural network, Continuous casting, Breakout prediction

Abstract. Aiming at the two terrible drawbacks of slow convergence and local optimal solution in the training process of BP neural network, particle swarm optimization algorithm was introduced to the training process of the BP neural network to improve its converge property, so a PSO-BP neural network was established, and then it was introduced into the breakout prediction system. The PSO-BP breakout prediction neural network model was trained and tested with the historical data collected from a steel plant. The results show that the convergence rate of the PSO-BP neural network model is significantly improved comparing the traditional BP neural network, and the feasibility of the model is verified by the testing result with the accuracy rate of $96.39 \%$ and the prediction rate of $100 \%$.
\end{abstract}

\section{Introduction}

High casting speed is the core of high efficient continuous casting technology, but with the improvement of casting speed, the risk of leakage of steel will increase[1]. Breakout becoming the key factor for restricting the speed increase, so the development of the real-time, effective breakout prediction system, for the possibility of leakage steel feature recognition, early warning is a major means of preventing breakout accident [2]. In this paper, the Particle Swarm Optimization (PSO) algorithm is introduced into the BP neural network, to optimize the weights of BP neural network. Then PSO-BP model for the breakout prediction during the continuous casting of steel is presented and compared with BP neural network using the same date observed from a steel plant.

\section{The prediction principle of thermocouple temperature measurement method}

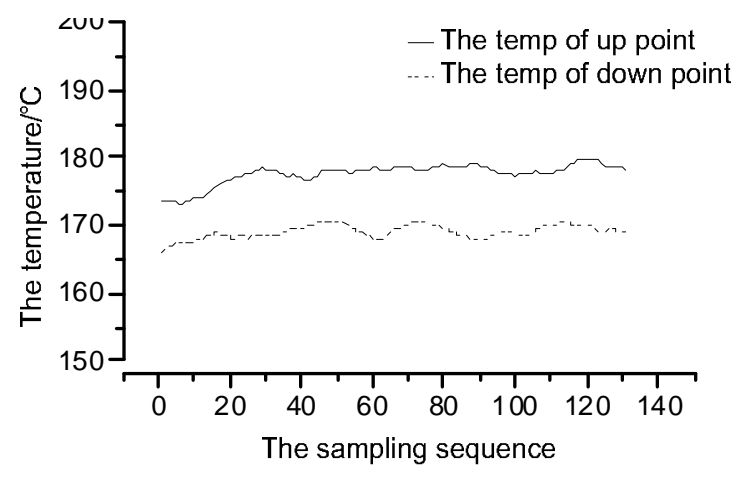

( a ) The normal temperature fluctuation

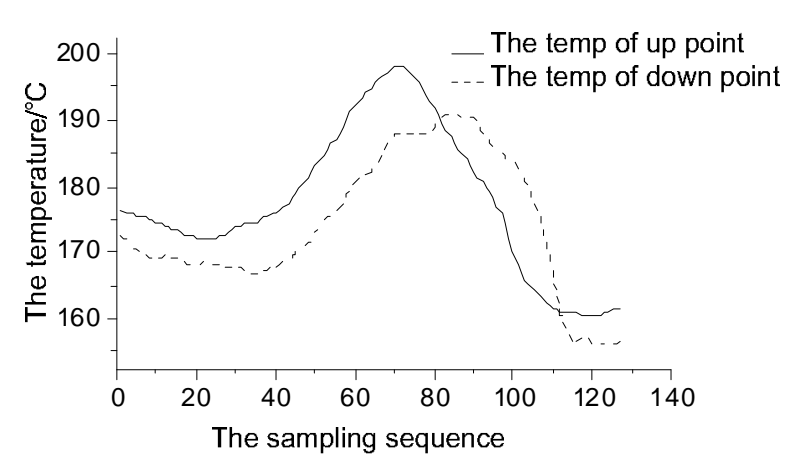

(b) The sticking temperature fluctuation

Fig. 1 The temperature variation modes in continuous casting progress

Adopted thermocouple temperature measurement method to forecast of leakage in the process of continuous casting steel, is likely to cause the temperature of the steel leakage model to make the right judgment, that is actually a kind of dynamic waveform pattern recognition problem, is to identify from the temperature of the detected waveform in steel leakage characteristics of waveform. The temperature variation modes in continuous casting progress shown in fig 1 . 


\section{The BP neural network}

In this paper, the structure of the BP neural network is the same as the standard BP network (see Fig.2).

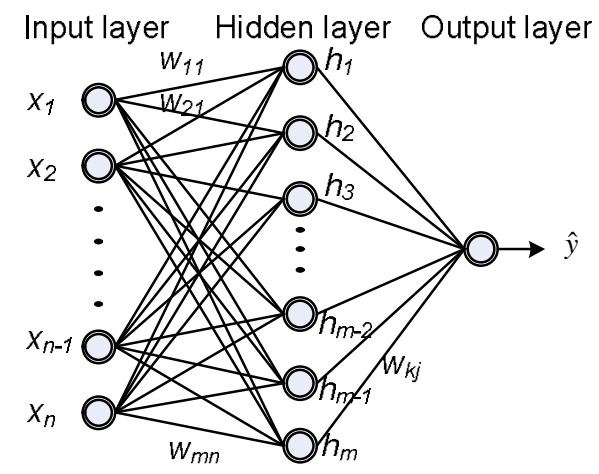

Fig. 2 The structure of the three layers' BP net

(1) Transfer function: According to the structure, the output of the BP neural network

$\hat{y}=\sum_{j}^{m} h_{j} w_{k j}+\theta_{k}$

where, $w_{k j}$ is the connection weights between input layer and hidden layer ; $\theta_{k}$ is the $\mathrm{k}$-th input layer neuron threshold value $; h_{j}$ is the value of the hidden nodes,

$$
h_{j}=\frac{2}{1+\exp \left(-2 *\left(\sum_{i=1}^{n} x_{i} w_{j i}+\theta_{j}\right)\right)}-1
$$

Where, $x_{i}$ is the input of the neural network ; $w_{j i}$ is the connection weights between the hidden layer and the output layer ; $\theta_{j}$ is the threshold value of the $\mathrm{j}$-th neuron of the hidden layer.

(2) The training error is the mean square error (MSE) of the neural network output, It is described as follows,

$M S E=\frac{1}{2} \sum_{p=1}^{N}\left(y_{p}-y_{p}^{\prime}\right)^{2}$

Where, $N$ is the number of training samples, $\mathrm{y}^{\prime}$ is the actual output, $\mathrm{y}$ is the desired output.

(3) Hidden layer nodes: According to Kolmogorov theorem, three-layer neural network approximate relationship between the input layer and the hidden layer nodes between the nodes :

$m \leq 2 n+1$

Where, $m$ is the number of hidden nodes, $n$ is the number of input layer nodes. Through trial and error, analysis, comparison verification results, and ultimately determine the hidden layer nodes is 28 .

\section{PSO-BP neural network model}

In this paper, the PSO is applied to the training process of the BP neural network, that is using the global particle swarm optimization algorithm to optimize the performance of BP neural network weights. The optimization process is as follows:

For the BP neural network showed in Fig. 2, set $W=\left\{W^{1}, W^{2}\right\}$

$$
W^{1}=\left[\begin{array}{ccc}
\omega_{11} & \mathrm{~K} & \omega_{1 n} \\
\mathrm{M} & \mathrm{O} & \mathrm{M} \\
\omega_{m 1} & \mathrm{~K} & \omega_{m n}
\end{array}\right], W^{2}=\left[\begin{array}{lll}
\omega_{1} & \mathrm{~K} & \omega_{m}
\end{array}\right]
$$

In the training process of the PSO-BP neural network, the i-th particle can be expressed as $W_{i}=\left\{W_{i}{ }^{1}, W_{i}{ }^{2}\right\}$, The best location for the i-th particle is $P_{i}=\left\{P_{i}{ }^{1}, P_{i}{ }^{2}\right\}$, Optimal location for the entire 
particle swarm $P_{g}=\left\{P_{g}{ }^{1}, P_{g}{ }^{2}\right\}$, The rate of position change of the i-th particle is $V_{i}=\left\{V_{i}{ }^{1}, V_{i}{ }^{2}\right\}$, The particle velocity and location update, respectively :

$$
V_{i}[t+1]=\alpha V_{i}[t]+c_{1} \varphi_{1}\left(P_{i}-W_{i}\right)+c_{2} \varphi_{2}\left(P_{g}-W_{i}\right)
$$

where, $\alpha$ is inertia weight ; $c_{1}$ is article tracking the history of the optimal value of the right to self-weight coefficient, $\mathrm{c}_{1}=2 ; \mathrm{c}_{2}$ is the rights for the optimal value of the particle tracking group weight coefficients, $c_{2}=2 ; \varphi_{1}, \varphi_{2}$ are random numbers within $[0,2]$;

$W_{i}[t+1]=W_{i}[t]+r V_{i}[t+1]$

Where, $r$ is the speed constraint factor, $\mathrm{R}=0.6$.

The weights of the neural network are adjusted by updating the particle position and velocity continuously until it satisfies the fitness criteria. $f\left(W_{i}\right)$ is the fitness function, $f\left(W_{i}\right)=\operatorname{MSE}\left(W_{i}\right)$

The algorithm of PSO-BP neural network is shown in Fig 3.

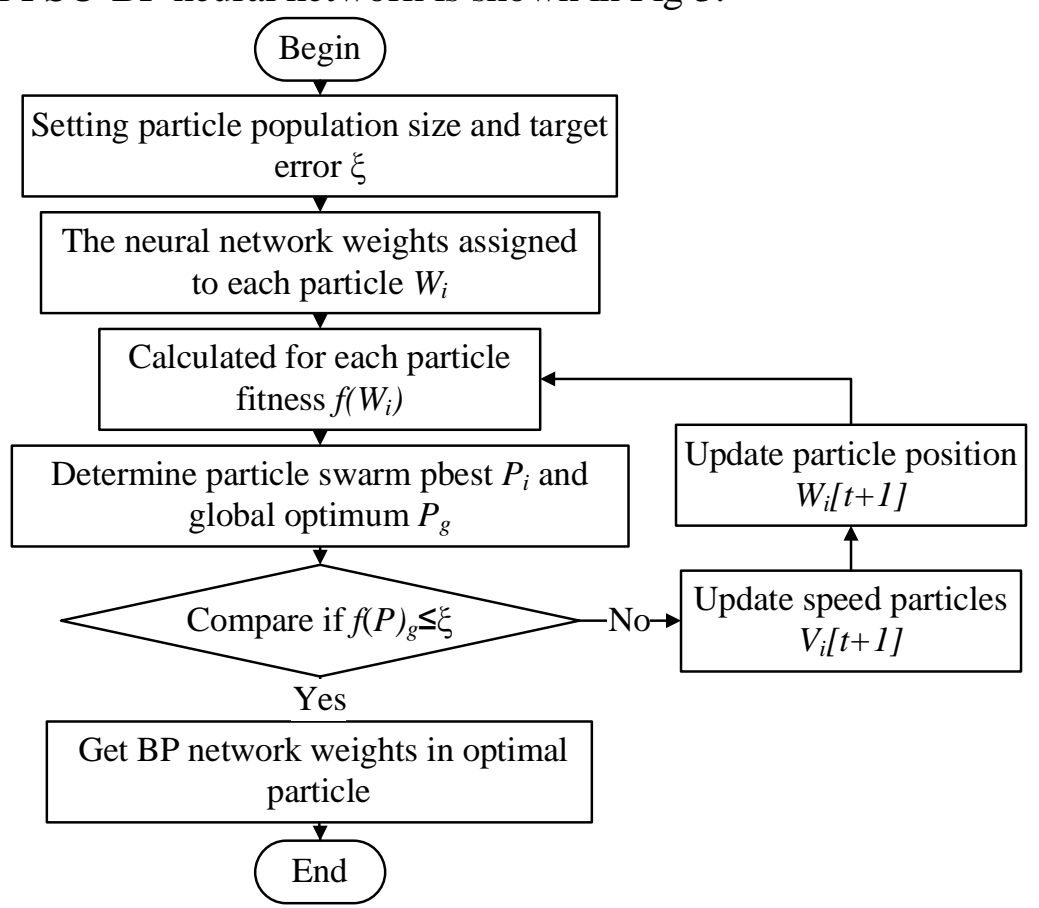

Fig. 3 The algorithm flow chart of the PSO-BP net

\section{Training and Testing of the breakout prediction model}

In order to eliminate the effects of the order of magnitude on the output of the neural network and highlight the date with Breakout features, the data are normalized as follows:

$$
X^{*}(i)= \begin{cases}\frac{X(i)}{\sqrt{\sum_{i=1}^{15} X^{2}(i)}} & \left(X_{\text {max }}-X_{\text {min }}\right) \leq \lambda \\ \frac{X(i)-X_{\text {min }}}{X_{\text {max }}-X_{\text {min }}} & \left(X_{\text {max }}-X_{\text {min }}\right)>\lambda\end{cases}
$$

Where, $X=\left\{X_{1}, X_{2}, \ldots X_{n}\right\}$ is the raw data, $n$ is the dimension of the input column vector, and $\lambda$ is the stability threshold.

The temperature sequence is considered to be stable, if the temperature change is not greater than $\lambda$. Otherwise, the temperature sequence is considered to be volatile. Usually, the change of the 
temperature is greater than $35^{\circ} \mathrm{C}$, when the breakout occurs. this paper takes $\lambda=25^{\circ} \mathrm{C}$ through the analysis of historical data collected in a steel plant.

In the continuous casting process, the smooth sequences of temperature account for the vast majority of the measurement. Using the general data normalization method would undermine this stability, and it is not conducive to the identification of the network model. So, the Eq. 8 is used for the data normalization in this paper, and that problem is well resolved.

A steel combined with historical data collected in the field from which to select a group of 200 sample sequence typical temperature pattern as the training sample PSO-BP neural network model, which includes 100 groups and 100 groups of samples stable pattern of breakout mode samples. Using the selected training samples respectively PSO-BP neural network model and BP neural network model of traditional training, the training results shown in the Fig. 4.

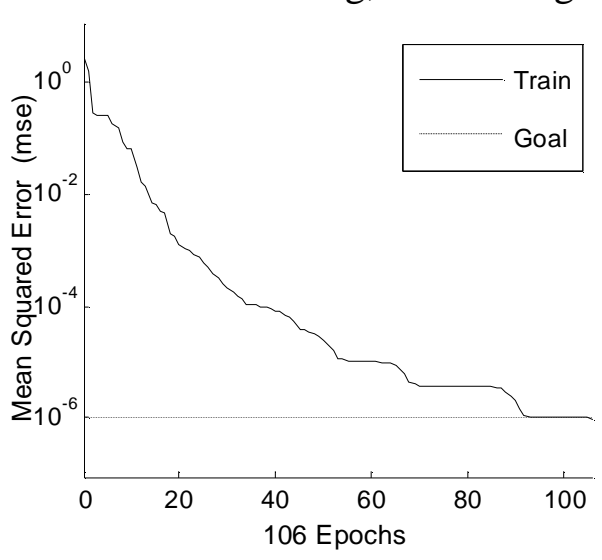

(a) The training process of PSO-BP model

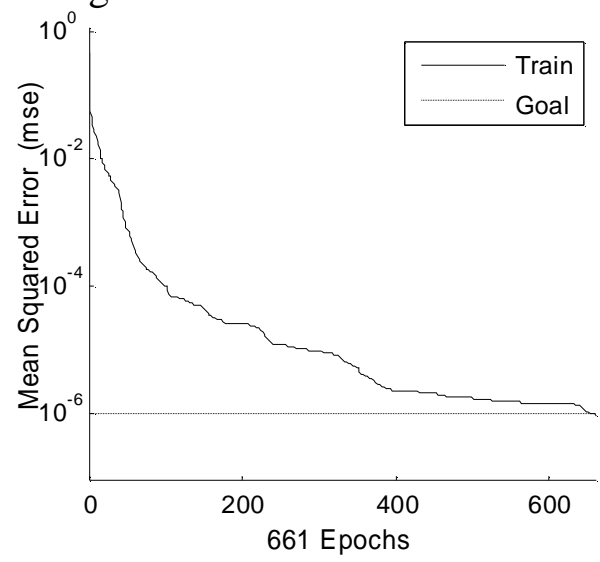

(b) The training process of BP model

Fig. 4 The training processes of the PSO-BP model and the BP model

The testing samples for the neural network models are 200 groups of typical temperature trace collected from the continuous casting plant, containing 80 groups of sticking type breakout samples, and 120 groups of normal samples. The result is shown in Table 1.

Table 1 The test results of the BP model and PSO-BP model

\begin{tabular}{|c|c|c|c|c|c|}
\hline & True & False & Omission & $\begin{array}{l}\text { Prediction } \\
\text { rate }\end{array}$ & $\begin{array}{c}\text { Accuracy } \\
\text { rate }\end{array}$ \\
\hline BP & $0^{8}$ & 5 & 0 & $100 \%$ & $\%^{94.12}$ \\
\hline $\mathrm{BP}^{\text {PSO- }}$ & $0^{8}$ & 3 & 0 & $100 \%$ & $\%$ \\
\hline
\end{tabular}

As can be seen from Table 1, two models have made the right judgments for all breakout samples, however, they both have false alarms; there are only 3 false alarms for the GA-BP neural network model, and the breakout prediction accuracy has been greatly improved.

\section{Conclusion}

This paper is designed PSO-BP neural network breakout prediction model, the nonlinear mapping ability and particle swarm optimization algorithm BP neural network of global optimization capability combine to overcome the traditional BP neural network convergence is slow and easy to fall into local minimum defects optimal solution. It will be applied to slab caster breakout prediction system, improve the accuracy of forecasting system on the bond breakout. Test results to predict the rate of $96.39 \%$ and $100 \%$ of the reported rate proved PSO-BP model feasibility and effectiveness of breakout forecasts.

\section{Acknowledgment}

The authors would like to acknowledge the support of Basic Research Program of Jiangsu province (No. BK20150429) and Yancheng Institute of Technology Talents Project(XJ201504). 


\section{References}

[1] Yibao Li, Xueyong Zhang, et al. Study of improving algorithms based on the BP neural network J. Journal of Hefei University of Technology, vol. 6 (2005), p. 668-671.

[2] Zhang Benguo, Zhang Ruizhong, Wang Ge, et al. Breakout prediction for continuous casting using genetic algorithm-based back propagation neural network model[J]. Int. J. Modeling, Identification and Control vol. 16(3) (2012), p. 199-205 